\title{
Spontaneous intestinal perforation followed by necrotizing enterocolitis in an extremely low birth weight neonate: case report and review of the literature
}

\author{
Ashraf H. M. Ibrahim", Hamad A. Hader ${ }^{1}$, Hatem Alsherbiny ${ }^{1}$, Mahmoud R. A. Hussein² and Safwan A. Khan
}

\begin{abstract}
Background: Spontaneous intestinal perforation (SIP) is usually an isolated perforation occurring most commonly in the terminal ileum of neonates with very low birth weight (VLBW) and extreme low birth weight (ELBW) of unknown etiology. It can be identical to perforated necrotizing enterocolitis (NEC) both clinically and radiologically raising a diagnostic challenge. In such cases, the differentiation is only possible by laparotomy and histopathology. This is important because the differentiation has management and prognostic consideration. The authors are presenting a case of SIP which was followed by NEC after 4 days only of its onset.

Case presentation: The authors report on a unique case of SIP which was followed by perforated NEC after 4 days of its onset in a 28-week gestational age with an ELBW. On the 3rd day of life, abdominal X-ray showed a significant pneumoperitoneum with neither pneumatosis intestinalis nor portal venous gas. The diagnosis of SIP was made at laparotomy. On the 4th post-operative day the patient developed pneumoperitoneum again due to perforated NEC as confirmed later on by histology. In this odd case (which we believe to be the youngest in the literature), the sequence of SIP followed after few days by perforated NEC is presented. Methods of differentiating these two conditions and their management are discussed.

Conclusion: SIP should be differentiated from perforated NEC by laparotomy and histopathology if this is not possible clinically and radiologically, and the patient is stable. Primary peritoneal drainage (PPD) is reserved as a step to stabilize the patient only in both conditions.
\end{abstract}

Keywords: $\mathrm{C}$ ase report, Spontaneous intestinal perforation, Necrotizing enterocolitis, Neonatal intestinal perforation

\section{Background}

The differentiation between SIP and perforated NEC based on clinical and radiological features can be difficult $[1,2]$. In such cases, the differentiation is only possible by laparotomy and histopathology. It is reported that PPD can be a definitive treatment of SIP without the need for laparotomy. This is only successful in $50 \%$ of the cases. Furthermore, no specimen for histology is

\footnotetext{
* Correspondence: ash_ib@hotmail.com

'Pediatric Surgery Department, Armed Forces Hospital Southern Region, P.O. Box 5062, King Faisal Military City, Saudi Arabia

Full list of author information is available at the end of the article
}

provided, the definitive diagnosis is never reached and proper treatment may have not been achieved. Our aim is to present an odd case of SIP followed by perforated NEC only 4 days after its onset. The methods of differentiating these two conditions and their treatment will be discussed.

\section{Case presentation}

After obtaining the ethical consent and consent for publication from the father, the authors present a 28-week gestational age preterm female baby. Her birth weight (BW) was $920 \mathrm{~g}$. She was delivered by an emergency cesarean section due to bradycardia. On admission, the 
baby was diagnosed as a case of respiratory distress syndrome with hypoxic ischemic encephalopathy, small ASD, and PDA. The patient was put on dopamine, kept nil per mouth with insertion of an umbilical venous catheter. There was thrombocytopenia $(50,000 / \mathrm{mL})$, INR was 3.1 , while C-reactive protein was 5 . Patient improved and was extubated. However, there was abdominal distension on the 3rd day of life. Abdominal X-ray showed a significant pneumoperitoneum with neither pneumatosis intestinalis nor portal venous gas (Fig. 1). With the provisional diagnosis of SIP vs perforated NEC, abdominal exploration was done. There was little amount of bilious staining which was negative for organisms according to culture sensitivity. The amount of the fluid was too little for chemical analysis. The stomach, duodenum, small, and large bowels were normal. This was interpreted as a case of SIP which had sealed. The abdomen was closed without a drain. Post-operatively, the patient was kept fasting with minimal aspirate via the nasogastric tube and the abdomen was soft and scaphoid till the 3rd post-operative day when she developed abdominal distension and thrombocytopenia (35, $000 / \mathrm{mL}$ ) but abdominal X-ray did not show pneumoperitonium. On 4th post-operative day she developed pneumoperitoneum again and the abdominal $\mathrm{X}$-ray was identical to Fig. 1. There was neither pneumatosis nor portal venous gas. The patient was optimized and reexplored. A single 7-mm-wide perforation was found about $40 \mathrm{~cm}$ proximal to ileocecal junction surrounded by an area of inflammation about $7 \mathrm{~cm}$ in length (NEC). The inflamed segment was resected with ileostomy performed. Histopathological examination of the resected part revealed a typical picture of NEC showing necrotic mucosa with loss of villus tip. The submucosa and the muscle layer showed evidence of coagulative with hemorrhagic necrosis with vascular thrombosis together with acute and chronic inflammatory cells (Fig. 2). The

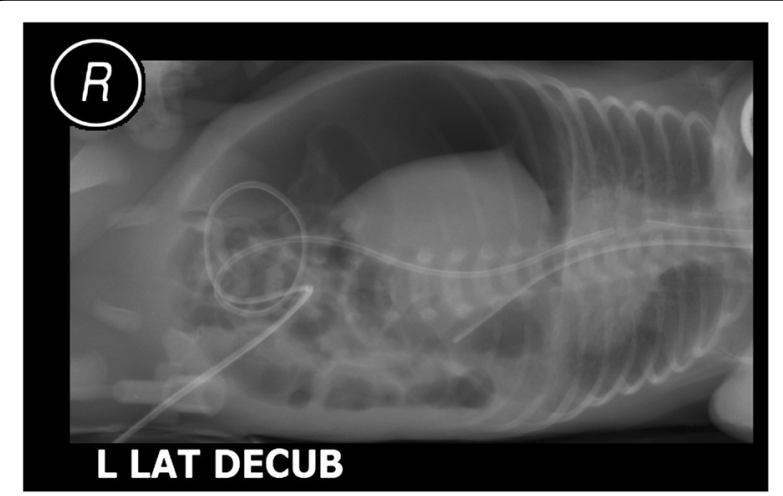

Fig. 1 A plain lateral decubitus $x$ ray of the abdomen showing massive pneumoperitonium with no evidence of pneumatosis intestinalis and no portal venous gas patient was gradually weaned off ventilator then off oxygen, kept nothing per mouth for 14 days, and then NGT feeding was tolerated but with poor weight gain.

\section{Discussion}

The main risk factors for SIP are prematurity, VLBW, ELBW, stress, hypoxia, shock, premature rupture of membranes, umbilical catheterization, and indomethacin therapy. Congenital defects of the intestinal musculature is also a well-known risk factor for SIP [3-5]. Many of these factors are present in this reported case. The tools for differentiation between SIP and NEC are clinical, radiological examination, exploratory laparotomy and confirmation by histology $[1,2]$. In this odd case (which we believe to be the youngest in the literature), the sequence of SIP followed by perforated NEC was present. SIP occurred in the first week while NEC perforation occurred in the second. Both conditions were identical clinically and radiologically and both showed the same picture of pneumoperitonium without the specific radiological signs of NEC. Upon exploration, SIP showed evidence of sealed perforation and completely normal stomach, small and large bowel (no histopathology specimens). Perforated NEC showed a 7 centimeters NEC affected segment with a 7-mm-wide perforation $40 \mathrm{~cm}$ proximal to the ileocecal junction. NEC perforation occurs due to coagulative necrosis which is unlikely to seal within few hours. It is possible for a tiny or small perforation to seal in a case of SIP. In contrast to NEC, histopathologic picture of SIP may show focal hemorrhagic necrosis, candida invasion, congenital defects with hypoplasia of the muscularis layer and thinning of the vessels in the submucosa $[6,7]$. The histology in SIP shows normal mucosa and muscularis mucosa with high villi. Around the perforation, the mucosa and submucosa are congested, edematous with bloody infiltration. In the perforated area, the muscularis propria may be absent and no fibrosis nor thrombotic phenomena [3-5]. In our case, there was a mixed histopathological picture of coagulative necrosis and hemorrhagic necrosis co-existing together. Clinical and radiological pictures were not enough to differentiate between the two conditions. Hence, laparotomy and histopathologic examination were the tools to confirm the diagnosis [2]. One case was reported in 1992 to have the two events of SIP at 2 days of age followed by NEC several weeks later when the case developed a typical clinical, radiological, and histological picture of NEC [4].

PPD can be used as a definitive treatment in stable patients of SIP without need for laparotomy [8-11]. The disadvantages of this conception is that it is successful at best in only $50 \%$ of patients [10], definitive diagnosis is never confirmed, proper management is not achieved and the golden hours for laparotomy may be missed. We 

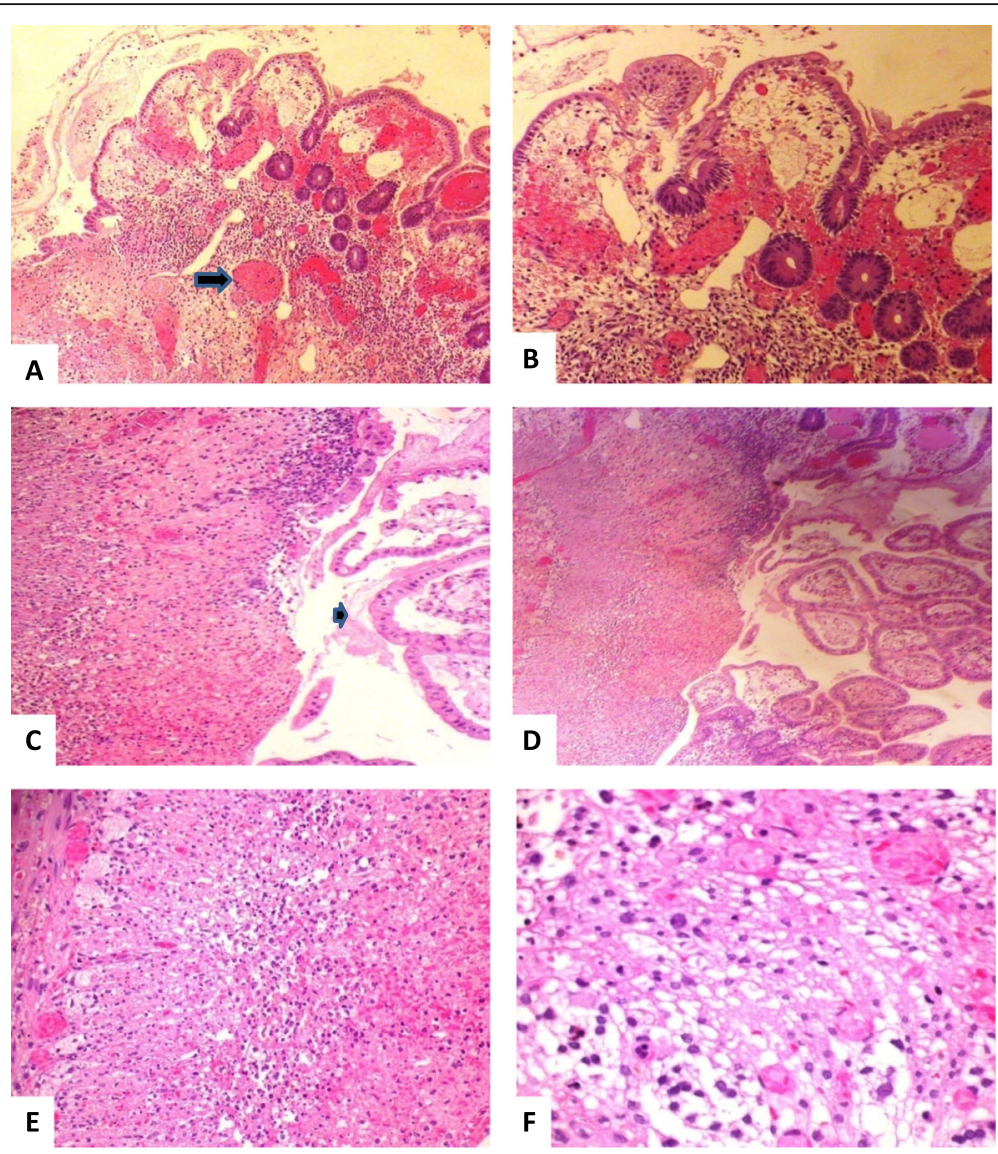

Fig. 2 a-f Histopathological changes in the small bowel showing various stages of intestinal injury. a, b There is disruption of the mucosal architecture (necrotic/degenerated materials on the mucosal side, loss of villus tip architecture, edema/hyperemia of the lamina propria, dilated lymphatics, and thrombus formation (arrow, $\mathbf{a}, \mathbf{b}$ ). c-f The bowel is affected much more severely. There is necrosis of the mucosa and submucosa with intraluminal-degenerated materials on the mucosal side of the bowel wall (arrowhead), coagulative, and hemorrhagic necrosis of the submucosa and muscle layers (d, arrow), associated with mixed inflammatory cell infiltrate including neutrophils, lymphocytes, and histiocytes (e, $\mathbf{f}$ arrow) and vascular thrombosis (e, arrowhead) and muscle layers (d, arrow), associated with mixed inflammatory cell infiltrate including neutrophils, lymphocytes and histiocytes (e, $\mathbf{f}$, arrow), and vascular thrombosis (e, arrowhead)

believe that PPD is indicated only in unstable patients as a step for stabilization for laparotomy, proper surgical procedure and histological sampling.

In a case of perforated NEC, the authors believe that resection and stoma formation is the best option since the level of NEC extension into the bowel can be difficult to assign during surgery and the possibility of a delayed distal stricture to develop is always present. A water soluble distal loopogram is mandatory before stoma closure 2-3 months later. After SIP, the prognosis is usually better than perforated NEC. Primary closure of the perforation or resection and primary anastomosis is a well-accepted option. Postoperative feeding can be started earlier than NEC perforation, the stoma can be closed earlier, and distal stricture is unlikely [1].

\section{Conclusion}

SIP should be differentiated from perforated NEC by laparotomy and histopathology if the patient is stable and clinical or radiological pictures are not conclusive. PPD can be used as a step to stabilize the patient only in both conditions.

\section{Abbreviations}

SIP: Spontaneous intestinal perforation; B.W: Birth weight; PPD: Primary peritoneal drainage; NGT: Nasogastric tube; NEC: Necrotizing enterocolitis; VLBW: Very low birth weight; ELBW: Extreme low birth weight

\section{Acknowledgements}

Not applicable

\section{Authors' contributions}

A.l is the senior operating surgeon and wrote the manuscript. H.H is the assisting surgeon, HA and SK made the literature review. M.H made the histopathological work. All authors have agreed to authorship and order of authorship. All authors have read and approved the manuscript.

\section{Funding}

None exists

\section{Availability of data and materials}

The data used in the current study are available from the corresponding author on reasonable request. 


\section{Ethics approval and consent to participate}

The Armed Forces Hospital Southern Region, Saudi Arabia Ethical Committee approved the case report and usage of the clinical information for research purposes after verbal informed consent ( AFHSRMREC/2019/pediatric

surgery/418)

\section{Consent for publication}

Father had consent for publication of patient clinical data and material.

\section{Competing interests}

The authors declare that they have no competing interests.

\section{Author details}

${ }^{1}$ Pediatric Surgery Department, Armed Forces Hospital Southern Region, P.O. Box 5062, King Faisal Military City, Saudi Arabia. ${ }^{2}$ Histopathology Department, Armed Forces Hospital Southern Region, P.O. Box 5062, King Faisal Military

City, Saudi Arabia.

Received: 11 December 2019 Accepted: 15 May 2020

Published online: 16 June 2020

\section{References}

1. Ibrahim AHM, Al-Hifzi I, Rashid K, Musalam AO, Al Ghamdi AA, El Hakeem H. Spontaneous Intestinal Perforation in Neonates. Ann Pediatric Surg. 2007;3: 130-4.

2. Shah J, Singhal N, da Silva O, Rouvinez-Bouali N, Seshia M, Lee SK, Shah PS. Intestinal perforation in very preterm neonates: risk factors and outcomes. J Perinatol. 2015;35(8):595-600.

3. Litwin A, Avidor R, Schujman E, et al. Neonatal Intestinal Perforation Caused by Congenital Defects of the Intestinal Musculature. Am J Clin Pathol. 1984; 81(1):77-80.

4. Izraeli $S$, Freud E, Mor C, et al. Neonatal intestinal perforation due to congenital defects in the intestinal muscularis. Eur J Pediatr. 1992;151(4): 300-3.

5. Dzienieka M, Grzelak-Krzymyanowska, Kulig A. Segmental congenital defects of the intestine musculature. Pol J Pathol. 2010;2:94-6.

6. Kubota A, Yamanaka H, Okuyama H, et al. Focal intestinal perforation in extremely-low-birth-weight neonates: etiological consideration from histological findings. Pediatr Surg Int. 2007;23:997.

7. Pumberger W, Mayr M, Kohl Hauser C, Weninger M. Spontaneous localized intestinal perforation in very-low-birth-weight infants: a distinct clinical entity different from necrotizing enterocolitis. J Am Coll Surg. 2002;195:796803.

8. Demestre $X$, Ginovart G, Figueras-Aloy J, et al. Peritoneal drainage as primary management in necrotizing enterocolitis: A prospective study. J Pediatr Surg. 2002;37:963-7.

9. Cass DL, Brandt ML, Patel DL, Nuchtern JG, Minifee PK, Wesson DE. Peritoneal drainage as definitive treatment for neonates with isolated intestinal perforation. J Pediatr Surg. 2000;35(11):1531-6.

10. Meier $D E$, Ahmeti M. Peritoneal drainage for newborn intestinal perforation: primary treatment or unnecessary delay? Ann Pediatric Surg. 2013;9:54-7.

11. Ye N, Yuan $Y, X u L$, Pfister RE, Yang C. Successful conservative treatment of intestinal perforation in VLBW and ELBW neonates: a single centre case series and review of the literature. BMC Pediatr. 2019;19:255.

\section{Publisher's Note}

Springer Nature remains neutral with regard to jurisdictional claims in published maps and institutional affiliations.

\section{Submit your manuscript to a SpringerOpen ${ }^{\circ}$ journal and benefit from:}

- Convenient online submission

- Rigorous peer review

- Open access: articles freely available online

- High visibility within the field

- Retaining the copyright to your article

Submit your next manuscript at $\boldsymbol{\nabla}$ springeropen.com 